\title{
Vergleichende Bestimmung von Testosteron im menschlichen Urin mittels Gaschromatographie respektive kompetitiver Proteinbindungsanalyse
}

\author{
Von K.-P. Littmann und H. Gerdes \\ Aus der Mediziniscben Universitätsklinik (Direktor: Prof. Dr. G. A. Martini) Marburg/Labn
}

(Eingegangen am 14. März/22. Mai 1973)

\begin{abstract}
Zwei Methoden zur Testosteronbestimmung im menschlichen Urin, die auf dem Prinzip der Gaschromatographie bzw. der kompetitiven Proteinbindungsanalyse beruhen, wurden zur Beurteilung ihrer L.cistungsfähigkeit im klinischen Bercich an einem einheitlichen Untersuchungsgut miteinander verglichen. Die Testosteronausscheidung wurde bei gesunden Männern und Frauen sowic Patienten mit verschiedenen Krankheitsbildern vor und nach I.eydigzell-Stimulation mit $3 \therefore 5000$ I. E. IICG gemessen.

Die Protcinbindungsmethode lieferte auch unter Berücksichtigung der unspezifischen Lecrwerte signifikant höhere Werte als dic Gaschromatographie. Der Korrelationskocffizient beider Mcthoden betrug r =:: 0,976. Dic Genauigkeit der Bestimmungen war größer bei der Gaschromatographic. Die Wiederfindungsmessungen mit markicrtem Testosterun ergaben für dic Gaschromatographic cinen Aufarbeitungsverlust von $17 \%$, für die Proteinbindungsanalyse von 31\%. Größere Spezifität, besscrc Reproduzicrbarkeit und größere Genauigkeit sprechen für eine Bevorzugung der gaschromatographischen Methode.
\end{abstract}

\section{Determination of urinary testosterone in man - a comparison between two methods using gas phase chromatography" and competitive protein binding analysis respectively}

Two methods for the determination of urinary testosterone in man based on gas phase chromatography and protein binding analysis respectively were compared in order to cvaluate their efficiency under clinical conditions. The 24 -hour testosterone excretion was measured in normal males and females and in patients with different diseases before and after Leydig ceil stimulation with $3 \times 5000 \mathrm{I}$. U. HCG. Individual values of testosterone excretion were significantly higher with the protcin binding analysis even taking into account the non specific blank values. The correlation coefficient of both methods was $r=0.976$. Differences exist in the accuracy. The loss of labeled testosterone during the whole analytic procedure was $170_{0}^{\circ}$ in gas chromatography and $31^{\circ} \%$ in protein binding analysis. The analysis by gas phase chromatography is preferable on account of its higher specificity, reproducibility and accuracy.

Seit der Isolierung von Testosteron aus menschlichem Urin von Schubert und WeHRBERGER 1960 (1) sind zahlreiche quantitative Bestimmungsmethoden entwickelt worden, die sich vor allem hinsichtlich des Arbeitsaufwandes, der Spezifität, der Empfindlichkeit, der Reproduzierbarkeit und der Präzision unterscheiden. Spektrophotometrische Testosteronbestimmungen von Caniacho und Migeon 1963 (2), Vermeulen und Verplancke 1963 (3), Voigt et al. 1964 (4) u. a. liefern insbesondere in niedrigen Konzentrationsbereichen keine ausreichend genauen Ergebnisse. Das Gleiche gilt für fluorimetrische Verfahren (5). Darüber hinaus werden diese Methoden als unspezifisch angesehen, weil sie eine sichere Differenzierung zwischen Testosteron und z. B. Epitestosteron, das ebenfalls im menschlichen Urin nachgewiesen wurde $(6,7)$, nicht ohne weiteres ermöglichen.

Für die Beantwortung klinischer Fragestellungen stehen gegenwärtig zwei Methoden zur Diskussion:

1. Die kompetitive Proteinbindungsanalyse (zuerst zur Plasmatestosteronbestimmung (Übersicht siehe bei NIESCHLAG et al. (8)).

2. Die gaschromatographische Testosteronbestimmung.

Im folgenden werden eine kompetitive Proteinbindungsmethode mit geringen Modifikationen nach UETTIVILLER (9) und eine gaschromatographische Methode nach Currius et al. (10) - wie sie in dieser Zeitschrift mit- geteilt wurden - bei gesunden Kontrollpersonen und bei Patienten mit verschiedenen Krankheitsbildern unter Ruhebedingungen und nach Leydigzell-Stimulation durch Gonadotropine verglichen.

\section{Methodik}

Untersuchungsmaterial

Urinproben wurden von 5 gesunden Männern im Alter von 16 bis 62 Jahren, von 3 Patienten mit Pubertas tarda im Alter von 14 bis 17 Jahren, von einer Patientin mit Hirsutismus im Alter von 35 Jahren, von 4 Patienten mit alkoholischer Lebercirrhose zwischen 45 und 66 Jahren und einem Patienten mit Anorchic im Alter von 23 Jahren gesammelt. Der Patient mit Anorchie hatte 3 Wochen zuvor zuletzt Testoviron Depot $250 \mathrm{mg}$ i. $\mathrm{m}$. (Fa. Schering) injiziert bekommen. Die Urinkollektion erfolgte bei allen Probanden unter Ruhebedingungen und am 4. Tag nach einer 3tägigen i. m.-Injektion von jeweils $5000 \mathrm{E} \mathrm{HCG}$ (Primogonyl, $\mathrm{Fa}$. Schering). Mehrfachbestimmungen wurden außerdem an einem Pool-Urin von 5 gesunden Männern vor und nach LeydigzellStimulation und an einem Pool-Urin gesunder Frauen durchgeführt.

\section{Arbeitsvorschriften}

Im folgenden werden nur die wichtigsten Aufarbeitungsschritte und die eigenen, geringfügigen Modifikationen beschrieben. Die Aufarbeitungsgänge sind original ausführlich mit Angabe der verwendeten Gerätc und Chemikalien in dieser Zeitschrift von Curtius et al. (10) und Uetrwiller (9) dargestellt.

\section{Gaschromatograplbische A. Fethode}

Je $100 \mathrm{ml}$ Utin werden mit 300 Fishman-Einheiten $\beta$-Glucuronidase pro $\mathrm{ml}$ bei pH 4,6 und $37^{\circ} \mathrm{C}$ über 72 Stunden hydrolysicrt. Danach werden zur Wiederfindungsmessung $0,5 \mathrm{ml}\left[{ }^{3} \mathrm{H}\right]$ Testo- 
steron gelöst in $\AA$ thanol (spez. Aktivität $162 \mathrm{mCi} / \mathrm{mg} ; 0,4 \mu \mathrm{Ci}$ in $1 \mathrm{ml}$ Athanol) zugefügt. Unter diesen Hydrolysebedingungen ist anzunehmen, daß nur Testosteron-Glucuronid gemessen wird. Der Verlust durch Hydrolyse wird nicht erfaßt. Es folgt die Extraktion der Testosteronglucuronidfraktion mit $3 \times 1$ VolumenAnteil Äther. Nach Ausschütteln mit $\mathrm{NaOH}$ und Neutralwaschen mit $\mathrm{H}_{2} \mathrm{O}$ wird eine Aluminiumoxid-Säulenchromatographie durchgeführt (4 $\mathrm{g}$ desaktiviertes Aluminiumoxid). Nach Vorwaschen mit $5 \mathrm{ml}$ Benzol/Äthanol (Verhältnis $99,9 \mathrm{ml}+0,1 \mathrm{ml}$ ) und Elution mit $2 \times 10 \mathrm{ml}$ Benzol/Äthanol (Verhältnis jeweils $99,9 \mathrm{ml}$ $+0,1 \mathrm{ml})$ - dies sind die Fraktionen $1 \mathrm{a}$ und $1 \mathrm{~b}$ - erfolgt Elution mit $30 \mathrm{ml} \mathrm{Benzol} /$ Äthanol (Verhältnis $99,75 \mathrm{ml}+0,25 \mathrm{ml}$ ) - das ist die Fraktion $2 \mathrm{a}$-, schließlich nochmals mit $2 \times 15 \mathrm{ml} \mathrm{Benzol} /$ Äthanol (Verhältnis $99,75 \mathrm{ml}+0,25 \mathrm{ml}$ )-Fraktionen $2 \mathrm{~b}$ und $2 c-$. Die vereinigten Fraktionen $2 a$ und $2 b$ werden eingedampft und im System Chloroform/Äthylacetat $(20 \mathrm{ml}+10 \mathrm{ml})$ dünnschichtchromatographiert. Die Testosteronzone wird mit $2 \times 5 \mathrm{ml}$ Äther eluiert, das Eluat zur Trockne eingedampft und anschließend in $10 \mathrm{ml}$ Äthylacetat aufgenommen. Davon werden $1 \mathrm{ml}$ zur $\left[{ }^{3} \mathrm{H}\right]$ Testosteron-Wiederfindungsmessung im Scintillationcounter (Fa. Packard) abgetrennt. Von den restlichen $9 \mathrm{ml}$ werden $4 \mathrm{ml}$ mit Stigmasterin als internem Standard ( $4 \mu \mathrm{g}$ Stigmasterin zu jeder Probe) und $4 \mathrm{ml}$ ohne internen Standard nach Bildung der Trimethylsilyl-Äther eingedampft, jeweils in $20 \mu \mathrm{l}$ Hexan wieder aufgenommen und zur Gaschromatographie gegeben.

Die Gaschromatographie erfolgte mit einem Gerät der Fa. Varian Typ Nr. 2100 mit Flammenionisationsdetektor auf 3\% XE 60 Säulen $6 \mathrm{ft} \times 1 / 4^{\prime \prime}$. Temperaturen: Injektor $225^{\circ} \mathrm{C}$, Säule $210^{\circ} \mathrm{C}$, Detektor $230^{\circ} \mathrm{C}$. Trügergas $\mathrm{N}_{2}=50 \mathrm{ml} / \mathrm{min}$. Die Auswertung der Peaks erfolgte mit einem Digitalintegrator. Die quantitative Berechnung von Testosteron geschah nach der von Curtrus et al. (10) angegebenen Methode; Abbildung 1 zeigt die Trennung von Epitestosteron, Testosteron und Stigmasterin zur Prüfung der Spezifität der Methode; Abbildung 2 zeigt ein Chromatogramm mit einer Urinprobe ohne Stigmasterin-Standard.

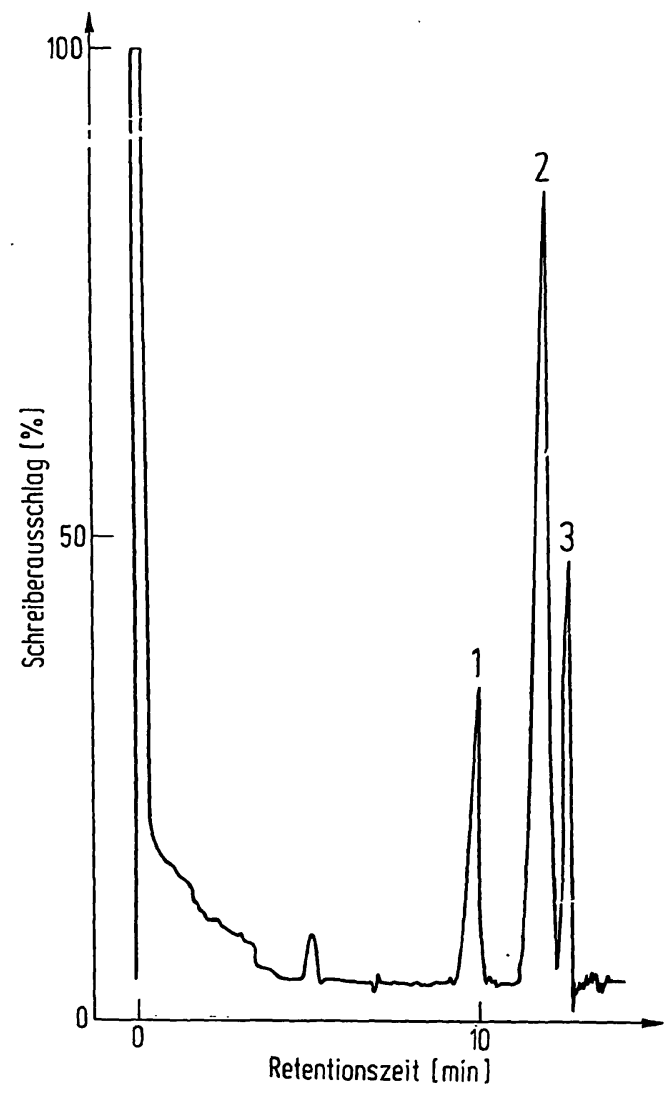

Abb. 1

Gaschromatogramm eines Testgemisches mit Rein-Substanzen von Epitestosteron (1), Testosteron (2) und Stigmasterin (3). Analyse-

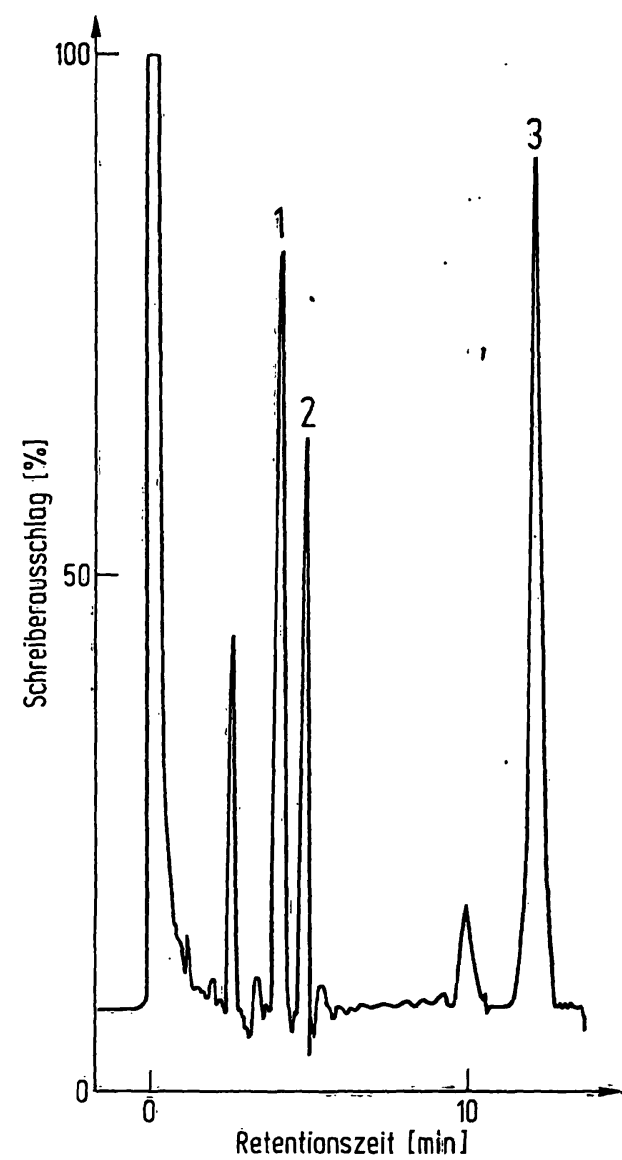

Abb. 2 Gaschromatogramim einer Urinprobe mit Androsteron (1), Atio-
cholanolon (2) und Testosteron (3). Analysebedingungen wie Abb.1

\section{Kompetitive Proteinbindungsanalyse}

Nach Hydrolyse von je $10 \mathrm{ml}$ Urin mit $1 \mathrm{ml}$ konzentrierter $\mathrm{H}_{2} \mathrm{SO}_{4}$ bei $90^{\circ} \mathrm{C}$ über $10 \mathrm{~min}$ wird mit $2 \times 50 \mathrm{ml}$ Äther extrahiert. Bei dieser Hydrolyse wird nicht nur glucuronidgekoppeltes, sondern auch sulfatverestertes Testosteron gespalten. Wiederfindungsversuche zur Berechnung des Substanzverlustes während der Aufarbeitung wurden mit $\left[{ }^{14} \mathrm{C}\right]$ Testosteron (spez. Aktivität $173 \mu \mathrm{Ci}$ / $\mathrm{mg} ; 2 \mathrm{nCi}$ in $10 \mathrm{ml}$ dest. Wasser/Äthanol im Verhältnis $8 \mathrm{ml}+2 \mathrm{ml}$ gelöst) so durchgeführt, daß von der Extraktion bis zur Inkubation sämtliche Aufarbeitungsschritte parallel ausgeführt wurden. Bei UETrwiller (9) fehlen nähere Angaben zur Durchführung der Wiederfindungsanalysen. Statt der Inkubation erfolgte bei den Wiederfindungsmessungen die Impulsratenzählung im Scintillationcounter.

Die Ätherextrakte wurden mit $\mathrm{NaOH}$ ausgeschüttelt und mit $\mathrm{H}_{2} \mathrm{O}$ neutralgewaschen. Der Äther wird abgedampft und der getrocknete Rückstand in Benzol gelöst. Es folgt eine Säulenchromatographie auf Aluminiumoxid $(3 \mathrm{~g}$ ) mit $35 \mathrm{ml}$ Benzol. Das Eluat wird verworfen. Danach wird erneut mit $2 \times 35 \mathrm{ml} \mathrm{Benzol} /$ Äther (Verhältnis $60 \mathrm{ml}+40 \mathrm{ml}$ ) eluiert. Die Benzol/Äther-Fräktionen werden eingedampft. Der getrocknete Rückstand wird in $100 \mu \mathrm{l}$ Methanol aufgenommen, von denen je $2 \times 20 \mu \mathrm{l}$ auf Dünnschichtplatten aufgetragen werden (Adsorbens: Alox MN-G-UV ${ }_{254}$ ). Chromatographie im System Methylenchlorid/Athylacetat (Verhältnis $95 \mathrm{ml}+5 \mathrm{ml})$. Nach Elution erneute Dünnschichtchromatographie (Adsorbens: Kieselgel MN-GHR-UV ${ }_{254}$ ) im Fließßmittel Chloroform/Aceton (Verhältnis $80 \mathrm{ml}+20 \mathrm{ml}$ ). Die Testosteronzone der zweiten Dünnschichtchromatographie wird mit Athanol eluiert, eingedampft und mit Äthanol in Inkubationsgläschen überführt. Nach Eindampfen Zugabe von $2 \mathrm{ml}$ einer 0,5proż. Plasmalösung (bestehend aus: Schwangerenplasma der 34.-36. Woche in Phosphatpuffer im Verhältnis $0,1 \mathrm{ml}+19,9 \mathrm{ml}, \mathrm{pH} 7,4$; Phosphatpuffer $0,125 \mathrm{~mol} / 1$ aus $\mathrm{KH}_{2} \mathrm{PO}_{4}, \mathrm{Na}_{2} \mathrm{HPO}_{4}$; der Phosphatpuffer enthielt $0 ; 061 \mathrm{ng} / \mathrm{ml}\left[{ }^{3} \mathrm{H}\right]$ Testosteron, spez. Aktivität $162 \mathrm{mCi} / \mathrm{mg}$ ) und Inkubation bei Raumtemperatur über $60 \mathrm{~min}$. Nach Zusatz 


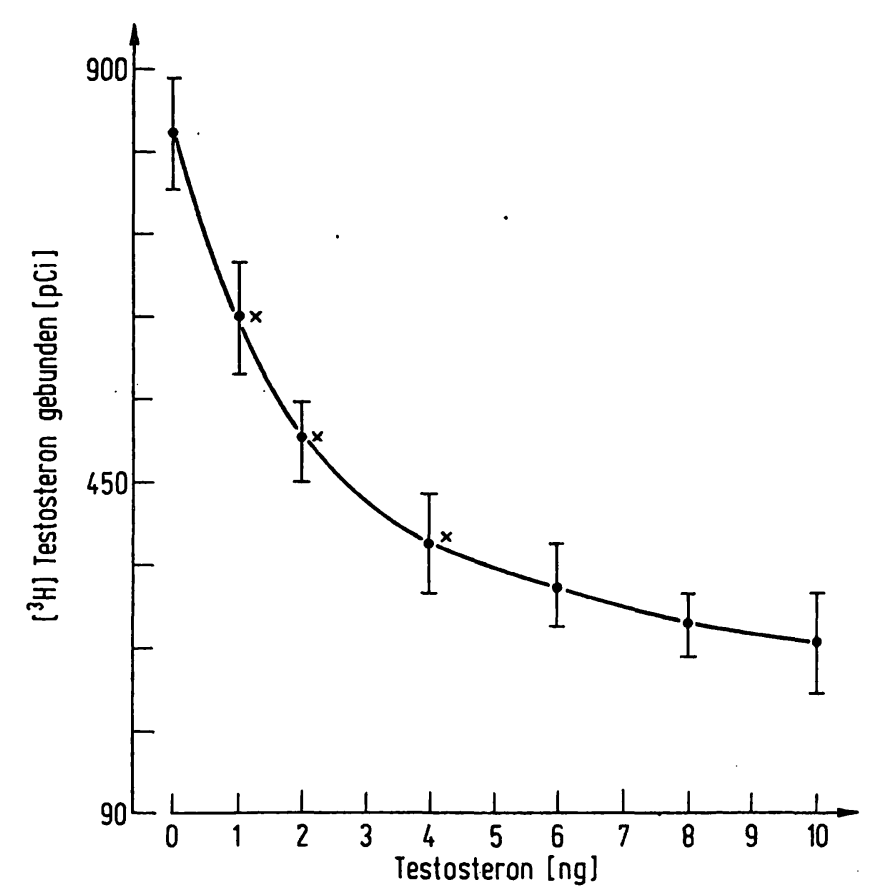

Abb. 3

Standardkurve für $0-10 \mathrm{ng}$ Testosteron mit der Protein-Bindungsanalyse. $n=8$. Mittelwerte mit Standardabweichungen. $x=$ Signifikanz $p<0,001$ gegenüber dem nächstkleineren Wert

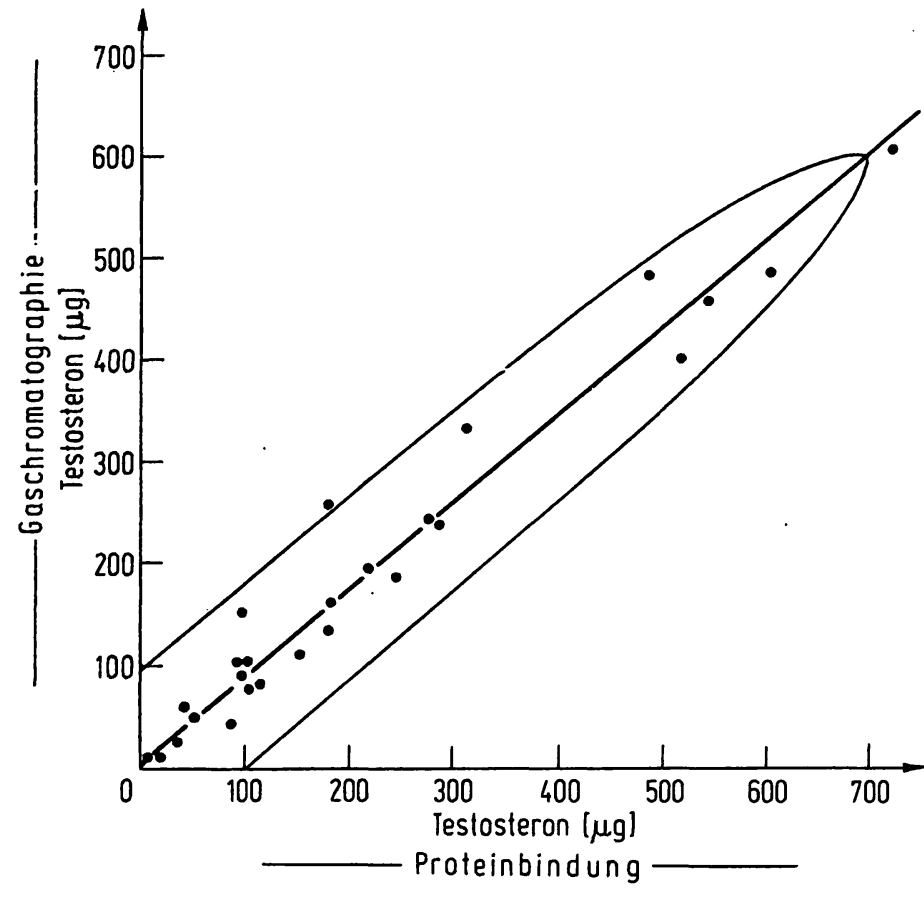

Abb. 4

Korrelationsgerade und Korrelationsellipse zweier Methoden zur Bestimmung von Testosteron im Urin. Errechnet nach den Ergebnissen von Tabelle 2. Die Korrelationsellipse enthält mit $95 \%$ iger Wahrscheinlichkeit alle tatsächlichen Meßpunkte

$$
r=0,976 \quad \operatorname{tg} \alpha=0,85
$$

von $80 \mathrm{mg}$ Florisil $1 \mathrm{~min}$ mechanisches Mischen, $5 \mathrm{~min}$ Stehenlassen; $900 \mu \mathrm{l}$ des Überstandes werden abpipettiert und die $\left[{ }^{3} \mathrm{H}\right]$ Testosteron-Aktivität dieser $900 \mu \mathrm{l}$ in $10 \mathrm{ml}$ Scintillationslösung (Aquasol, Fa. NEN, England) gemessen. Abbildung 3 zeigt die Eichkurve für Konzentrationen von 1 bis $10 \mathrm{ng}$ Testosteron.

\section{Ergebnisse}

Die 10-fach-Bestimmungen von Testosteron in einem Pool-Urin von gesunden Frauen und gesunden Männern vor und nach Leydigzell-Stimulation ergeben mit der Proteinbindungsanalyse in allen Fällen signifikant höhere Werte als mit der gaschromatographischen Methode (Tab. 1); Signifikanz $p<0,001$ im t-Test. Dabei ist die Genauigkeit der gaschromatographischen Methode sowohl bei niedrigen als auch bei hohen Urintestosteronkonzentrationen größer als die der Proteinbindungsanalyse. Auch die Wiederfindung markierten Testosterons am Ende des Aufarbeitungsganges zeigt

Tab. 1

Genauigkeit und Reproduzierbarkeit der Bestimmung von Testosteron im Urin mit zwei verschiedenen Methoden aus Pool-Urinen von gesunden Männern vor und nach Stimulation mit Humangonadotropin und Frauen. $\mathrm{n}=10$ Einzelbestimmungen. $\overline{\mathrm{x}}=$ Mittelwert; $\mathrm{s}=$ Standardabweichung; VK = Variationskoeffizient

\begin{tabular}{|c|c|c|c|c|c|c|}
\hline & \multicolumn{3}{|c|}{$\begin{array}{c}\text { Gaschromatographie } \\
{[\mu \mathrm{g} / 24 \mathrm{~h}]}\end{array}$} & \multicolumn{3}{|c|}{$\begin{array}{l}\text { Proteinbindungsanalyse } \\
{[\mu \mathrm{g} / 24 \mathrm{~h}]}\end{array}$} \\
\hline & $\overline{\mathbf{x}}$ & $\mathbf{s}$ & VK & $\overline{\mathbf{x}}$ & $\mathbf{s}$ & VK \\
\hline $\begin{array}{l}\text { Männer } \\
\text { vor } \mathrm{HCG} \\
\text { nach } \mathrm{HCG}\end{array}$ & $\begin{array}{l}106 \\
520\end{array}$ & $\begin{array}{l} \pm 5,0 \\
\pm 22\end{array}$ & $\begin{array}{l}4,7 \\
4,3\end{array}$ & $\begin{array}{l}131 \\
628\end{array}$ & $\begin{array}{l} \pm 11,2 \\
\pm 51\end{array}$ & $\begin{array}{l}8,5 \\
8,1\end{array}$ \\
\hline Frauen & \multicolumn{2}{|c|}{$8,9 \pm 0,5$} & 5,6 & \multicolumn{2}{|c|}{$12,4 \pm 1,2$} & 9,7 \\
\hline $\begin{array}{l}\text { Wiederfindung } \\
\text { alle Gruppen [\%] }\end{array}$ & 83 & \pm 8 & 9,7 & 69 & \pm 13 & 19,4 \\
\hline
\end{tabular}

für die gaschromatographische Methode deutlich bessere Werte. Beide Methoden korrelieren sehr gut (Abb. 4) sowohl im niedrigen Konzentrationsbereich (z. B. bei Patienten mit Anorchie, bei Frauen) als auch bei hohen Konzentrationen nach Gonadotropinstimulation gesunder Männer.

Für gesunde männliche Kontrollpersonen wurden Testosteronausscheidungen im 24-h-Urin zwischen 82 und $158 \mu \mathrm{g}$ mit der Gaschromatographie und zwischen 90 und $184 \mu \mathrm{g}$ mit der Proteinbindungsanalyse gemessen. Nach Stimulation mit Gonadotropin stiegen die Werte um den Faktor 3,9 bis 5,0 bei gaschromatographischen Messungen und um 3,5 bis 5,9 bei Messungen mit dem Proteinbindungsverfahren an (Tab. 2). Niedrigere Ausgangswerte, jedoch mit einem höheren Faktor des Anstiegs nach Gonadotropin hatten die Patienten mit einer konstitutionell verzögerten Pubertät. Die Leydigzell-Stimulierbarkeit bei 4 Patienten mit Lebercirrhose lag deutlich unter der gesunder Kontrollpersonen. Eine sehr niedrige Testosteronausscheidung wurde bei einem Patienten mit Anorchie gemessen, der mit Testoviron Depot substituiert war, und bei einer Patientin mit idiopathischem Hirsutismus ohne weitere Hinweise für ein adrenogenitales Syndrom. Die mit beiden Methoden gemessenen 24-h-Ausscheidungen von Testosteron stimmen mit den in der Literatur angegebenen Werten gut überein (11).

Die fünffache Bestimmung einer vorgegebenen Menge von $100 \mu \mathrm{g}$. Testosteron in dest. Wasser/Äthanol $90 \mathrm{ml}+10 \mathrm{ml} \mathrm{zu}$ Beginn der Analysen ergab mit der Gaschromatographie im Mittel $97 \pm 6,7 \mu \mathrm{g}$, mit der Proteinbindungsanalyse $95 \pm 11,9 \mu \mathrm{g}$. 
Tab. 2

Testosteronausscheidung im 24-h-Urin in $\mu \mathrm{g}$ bei Gesunden und Patienten mit verschiedenen Erkrankungen vor und nach Stimulation mit Humangonadotropin. Vergleich der mit der Gaschromatographie bzw. Proteinbindungsanalyse gewonnenen Werte. J. P. weiblich; alle anderen Probanden männlich

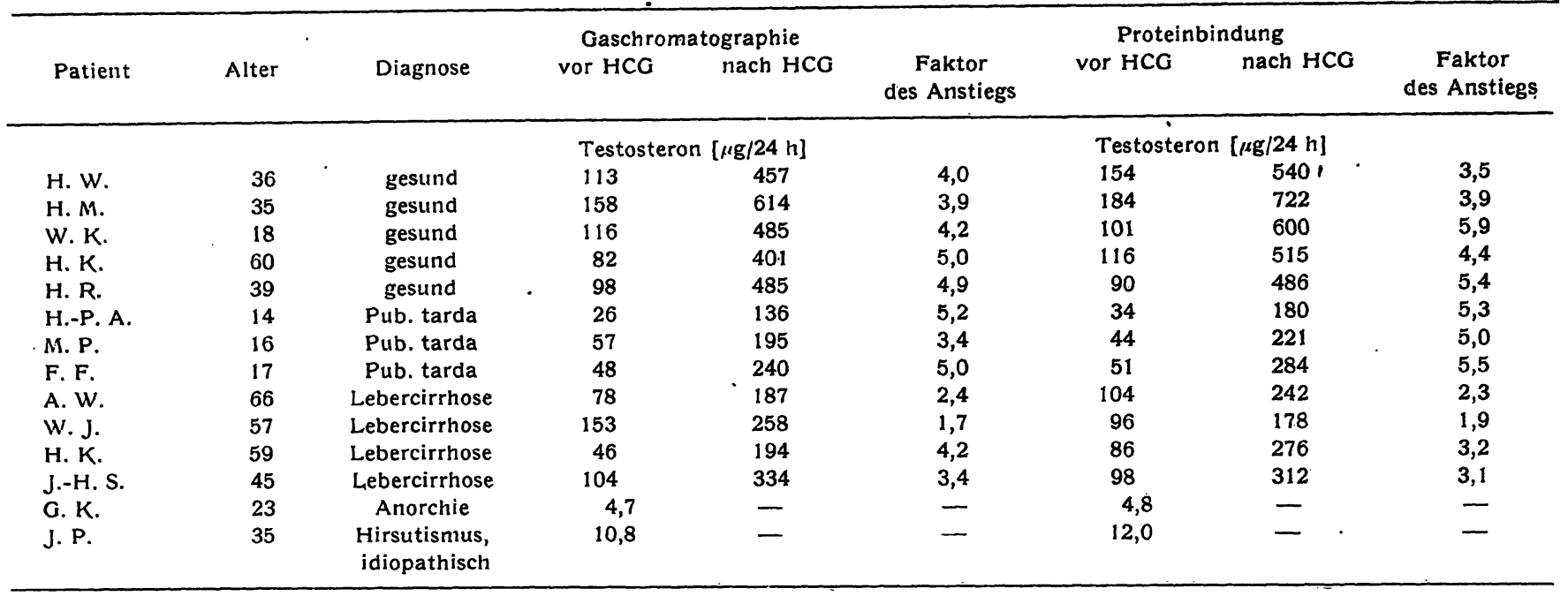

\section{Diskussion}

Eine gaschromatographische Methode und eine kompetitive Proteinbindungsmethode zur Bestimmung von Testosteron im menschlichen Urin wurden in Parallelbestimmungen an einem Kollektiv von Gesunden und Patienten mit verschiedenen Krankheitsbildern verglichen. Die Trennung von freiem und gebundenem $\left[{ }^{3} \mathrm{H}\right]$ Testosteron erfolgte bei der Proteinbindungsmethode mittels Florisil in einem Inkubationsverfahren, das einem Dialyseverfahren, einer Ultrafiltration oder einer Trennung an Sephadex oder Dextran-Aktivkohle wegen seiner besonders guten Trenneigenschaften vorgezogen wurde $(9,12)$. Die Gaschromatographie wurde auf 3\% XE 60-Säulen nach Bildung von Trimethylsilyläthern durchgeführt. Unter diesen Bedingungen ergibt sich eine günstige Trennleistung gegenüber anderen Steroiden und auch bei geringen Konzentrationen eine hohe Empfindlichkeit $(6,10,11,13-15)$.

Höhere Wiederfindungsraten und größere Genauigkeit liefert die gaschromatographische Methode. Eine Aussage über die Richtigkeit beider Methoden kann dagegen nur mit Einschränkung gemacht werden, da verschiedene $\mathrm{Hydrol}$ yseverfahren benutzt wurden. Wenn etwa $98 \%$ des im Urin ausgeschiedenen Testosterons an Glucuronsäure gekoppelt sind $(15,16)$ und die restlichen 1 bis $2 \%$ als freies Testosteron bzw. als Sulfatester ausgeschieden werden, ist von einer enzymatischen Hydrolyse - wie bei der Gaschromatographie - nicht zu erwarten, daß sie die „richtige“, also tatsächlich ausgeschiedenèe Gesamttestosteronmenge erfaßt. Die nach saurer $\mathrm{Hy}$ drolyse durchgeführte Urintestosteronbestimmung - wie bei der Proteinbindungsanalyse - müßte insgesamt höhere Werte liefern. Das erklärt jedoch nicht den von uns gefundenen Unterschied von etwa $20 \%$ höheren Werten mit der Proteinbindungsanalyse (Mịttelwerte, Tab. 1), die deutlich über dem zu erwartenden Bereich liegen (15).
Insgesamt scheinen mehrere Gründe für die Unterschiede in der Wiederfindung, der Genauigkeit und Richtigkeit verantwortlich zu sein:

1. Die Proteinbindungsanalyse erfordert eine zusätzliche Dünnschichtchromatographie zur Abtrennung von Epitestosteron. Bei jeder Dünnschichtchromatographie muß aber mit einem durchschnittlichen Substanzverlust von $7-10 \%$ gerechnet werden (9). Die Wiederfindung wird demzufolge um diesen Prozentsatz geringer werden. Nach den vorliegenden Untersuchungen liegt sie um etwa $14 \%$ niedriger als bei der Gaschromatographie. Der Fehler läßt sich durch die Anwendung des endogenen Standards ausgleichen.

2. Ein bislang noch ungelöstes Problem der kompetitiven Proteinbindungsanalyse sind die unspezifischen Leerwerte, deren Ursachen an anderer Stelle bereits ausführlich diskutiert wurden. Die von UETTwiLler (9) beschriebene Methode erfordert u. a. daher die Verwendung spektralreiner Lösungsmittel, die Waschung von Kieselgel und Aluminiumoxid mit Methanol und Äther und eine Dünnschicht-Eluierung der Standards, wie sie auch für die Urinproben durchgeführt wird. Dennoch ergeben sich Leerwerte von 0,2 bis $0,4 \mathrm{ng}$ Testosteron, die bei einer Messung der Einzelproben im Konzentrationsbereich von 3 bis $7 \mathrm{ng}$ Ungenauigkeiten zwischen 10 und $15 \%$ möglich machen.

3. Während mit der Gaschromatographie eine sichere Trennung des Testosterons von anderen $\mathrm{C}_{19}$-Steroiden gelingt, bleibt die Spezifität der Proteinbindungsanalyse trotz intensiver Vorreinigung und Vortrennungen unsicher. Möglicherweise ist dieses ein wesentlicher Grund dafür, daß die-Einzelmeßwerte in der überwiegenden Zahl höher liegen als mit der Gaschromatographie.

4. Mit der gaschromatographischen Endpunktbestimmung wird die Testosteronkonzentration im $\mu \mathrm{g}$-Bereich gemessen, mit der Proteinbindungsanalyse im ng-Bereich. Daş ergibt für die Proteinbindungsanalyse die 
Notwendigkeit, mit größeren Multiplikatoren auf die Gesamtausscheidung im 24-h-Urin umzurechnen. Auch dabei sind Fehlermöglichkeiten nicht auszuschließen.

5. Die Testosteronbindungskapazität, d. h. die Konzentration des spezifischen $\beta$-Globulins der Schwangerenseren ist qualitativ unterschiedlich. Die Verwendung verschiedener Chargen erfordert daher jeweils wieder eine neue, sehr zeitraubende Erarbeitung von Eichkurven.

6. Bei der hier im Vergleich untersuchten kompetitiven Proteinbindungsmethode erfolgte die Trennung des freien und proteingebundenen Testosterons nicht über Florisil-oder andere Säulen, sondern - aus Zeitersparnisgründen - mit Hilfe eines Florisilgemisches im Inkubationsgefäß. Auch dieses Vorgehen hat wahrscheinlich Nachteile - z. B. der Standardisierung wie sie bei Säulen möglich ist -, die den Ungenauigkeitsfaktor vergrößern.

Nach den vorliegenden Ergebnissen stellt die Proteinbindungsanalyse eine hochempfindliche Methode zur Testosteronbestimmung im Urin dar, die aber mit sehr viel mehr Störmöglichkeiten als die gaschromato- graphische Bestimmung zu rechnen hat. Beide Methoden besitzen eine gute Korrelation, so daß ihre klinische Anwendung gerechtfertigt ist. Beide Methoden erfordern jedoch gleichgroße labortechnische Erfahrung, die ihre Anwendung Speziallaboratorien vorbehält. Die wesentlichen Vorteile der Proteinbindungsanalyse liegen einmal in ihrer Unabhängigkeit von einer aufwendigen apparativen Laborausstattung, zum anderen in einer kürzeren Aufarbeitungsdauer der Proben - die vorwiegend durch den wenig arbeitsintensiven Schritt der Hydrolyse bedingt ist -, die im klinischen Routinegebrauch, etwa bei der Differentialdiagnose hypogonadotroper Syndrome, jedoch kaum von Bedeutung sein dürfte. Aufgrund ihrer größeren Genauigkeit und Reproduzierbarkeit ist die Gaschromatographie für Bilanzstudien geeigneter.

\section{Danksagung}

Herrn Prof. Dr. ScııaumlöııEı,, Klinik und Poliklinik für Nuklearmedizin der Universität Marburg, danken wir für die Hilfe bei den Radioaktivitätsmessungen. Frau L. Kopp danken wir für die ausgezeichnete technische Assisten\%. Die Arbeit wurde mit Unterstützung der Deutschen Forschungsgemeinschaft durchgeführt.

\section{Literatur}

1. SCHUbert, K. \& WeHrberger, K. (1960), Naturwissenschaften 47, 281. - 2. Camacho, A. M. \& Migeon, C. J. (1963), J. Clin. Endocrinol. Metab. 23, 301-305. - 3. VerMieuLeN, A. \& VerplanCKe, J. C. M. (1963), Steroids 2, 453-463. - 4. VoIGT, K. D., VOLKWEIN, U. \& TAMrM, J. (1964), Klin. Wochenschr. 42, 642-644. - 5. Graef, V., Jobst, P. \& Staudinger, HJ. (1968), diese Z. 6, 159-162. - 6. Futterweit, W., Siegel, G. L., Griboff, S. I., Dorfman, R. I. \& SOFFek, L. J. (1965), J. Clin. Endocrinol. Metab. 25, 1451-1456. - 7. Korenman, S. G., WiLson, H. \& Lipsetr, M. B. (1964), J. Biol. Chem. 239, 1004 bis 1006. - 8. Nieschlag, E., Rohr, M., Wombacher, H. \& Overzier, C. (1971), Klin. Wochenschr. 49, 91-100. - 9. Uetrwiller, A. (1970), diese Z. 8, 225-230. - 10. Curtius, H.-Ch., ZaCHMANN, M. \& MüLleR, M. (1970), diese Z. 8, 221 bis
224. - 11. Straib, W. (1968), In das Testosteron - Die Struma, (KLEIN, E., Hrsg.) S. 32-51, Springer Verlag, BerlinHeidelberg-New York. - 12. RudD, B. T., RosenfIELD, R. I., Bonglovanni, A. M. \& Eherlein, W. R. (1969), Steroids 13, 227-245. - 13. Futterweit, W., MCNiven, N. L., Narcus, L., Lantos, C., Drosdowsky, M. \& Dorfanan, R. I. (1963), Steroids 1, 628-642. - 14. Gibree, N. B., Forchielli, E., Strauss, J. S., Pocin, P. E. \& Dorfman, R. 1. (1965), Proc. Soc. Exp. Biol. Med. 119, 1019-1021. - 15. van der Molen, H. J., Groen, D. \& Peterse, A. (1966), In Androgens in normal and pathological conditions (Verareulen, A. \& Exley, D., eds.) 101, p. 1-10 Excerpta Medica Foundation Intern. Congr. Series. 16. Dulmanis, A., Coghlan, J. P., Wintour, M. \& Hudson, B. (1964), Aust. J. Exp. Biol. Med. Sci. 42, 385-400.

Dr. Klaus-Peter Littmann Med. Univ.-Klinik 3550 Marburg/Lahn Mannkopffstr. 1 